Research Paper

\title{
St Venant Torsion and Bending of Prismatic Composite Shafts
}

\author{
BHUSHAN LAL KARIHALOO ${ }^{1, *}$ AND QI-ZHI XIAO ${ }^{2}$ \\ ${ }^{1}$ School of Engineering, Cardiff University, Cardiff, CF24 3AA, UK \\ ${ }^{2}$ LUSAS FEA Ltd, Forge House, 66 High Street, Kingston-upon-Thames, KT1 1HN, UK
}

(Received on 20 April 2016; Accepted on 28 April 2016)

\begin{abstract}
This paper discusses the use of high-performance incompatible, enhanced-strain and hybrid stress elements for the St Venant torsion and bending of composite prismatic shafts, formulated in terms of the warping function. The properties of the shaft are uniform along its length but piece-wise constant and orthotropic across its cross-section. In order to analyse composite shafts whose microstructural details are difficult to be modelled directly by finite element discretisation, the two-scale asymptotic (or mathematical) homogenisation approach is also discussed; the resulting equilibrium equations can be readily solved by the aforementioned high-performance elements.
\end{abstract}

Keywords: Composite Shaft; Enhanced-strain Element; Homogenisation; Hybrid Stress Element; Incompatible Element; Bending; Torsion

\section{Introduction}

St Venant torsion and bending of prismatic shafts are fundamental problems in the theory of elasticity and of wide interest in engineering design, see e.g. Pilkey (2002). It is also an essential ingredient in the development of advanced (Timoshenko) beam theory with shear correction (Gruttmann and Wagner, 2001) and/or torsional warping (Battini and Pacoste, 2002; Gruttmann et al, 2000; Simo and Vu-Quoc, 1991).

Many useful analytical solutions for torsion and bending of isotropic homogeneous shafts have been presented in books on theory of elasticity, e.g. Timoshenko and Goodier (1969). Karihaloo and Hemp (1987) and Pilkey (2002) have discussed optimization of cross-sectional shapes of shafts. Most recently, Romano et al. (2012) revisited the St Venant beam theory under shear and torsion with special attention to the notions of shear and twist centres; they used Matlab to solve the boundary value problems for simple regions.

Development of finite element approaches for the analysis of St Venant torsion began almost at the same time as the finite element method itself. A detailed discussion of the various approaches can be found in Desai (1979). Many early finite elements are based on stresses or stress functions and are limited to simply connected cross-sections. Xiao et al. (1999) have introduced an optimised hybrid stress element; Karihaloo et al. (2001) have developed displacement-incompatible and enhanced-strain elements. These high-performance elements are applicable for simply as well as multiply connected cross-sections.

Since the pioneering work of Mason and Herrmann (1968) on the development of a triangular displacement element from the principle of minimum potential energy, many studies have been carried out on the finite element analysis of St Venant bending. Gruttmann et al. (1999) and Gruttmann and Wagner (2001) formulated St Venant bending in terms of the bending warping function, which can be solved in a similar manner to the torsional warping function. They introduced a displacement compatible element to solve for bending warping, and a method for computing the shear correction factors for Timoshenko beams with arbitrary cross-sections assuming uncoupling of the strain energy for bending and torsion, and equivalence

*Authorfor Correspondence: E-mail: karihaloob@cardiff.ac.uk 
of the strain energy from the average and equilibrium shear stresses. Jog and Mokashi (2014) studied bending of isotropic homogeneous bars with special attention to the torsion effect caused by the shearing forces that do not pass through the shear centre.

Lekhnitskii (1963) has explored torsion and bending of orthotropic homogeneous beams with simply connected cross-sections, making recourse to stress functions. Kosmatka and Dong (1991) studied prismatic anisotropic beams using the Ritz method based on the principle of minimum potential energy.

Kourtis et al. (2009) formulated compatible elements based on a combined warping function for torsion and transverse shearing of isotropic or transversely isotropic materials. They assumed that the elastic and shear moduli are spatial functions across the cross-section and considered the effects of their gradients; Poisson's ratio was assumed to be constant. With the use of Matlab to solve the boundary value problems for simple regions, Barretta (2013) studied orthotropic St Venant beams with a spatially constant Poisson tensor and fibre-wise homogeneous moduli of elasticity and shear with special attention on shear and twist centres. Jog and Mokashi (2014) studied torsion of prismatic compound anisotropic bars using 4- and 9-node compatible elements. Mokos and Sapountzakis (2005) also studied bending of composite beams with constant Poisson's ratio and piece-wise constant moduli of elasticity and shear by a boundary element method.

For composite shafts whose microstructural details are difficult to model directly using the finite element discretisation, Karihaloo et al. (2001) introduced the two-level asymptotic (or mathematical) homogenisation approach for periodic microstructures to the analysis of torsion. Yuan et al. (2003) used two-level homogenisation to the topology optimisation of cross-section for composite shafts under St Venant torsion.

This paper will discuss the solution of St Venant torsion and bending of prismatic composite shafts via high-performance finite elements or two-scale homogenisation. For St Venant bending, the violation of the assumption of zero in-plane shear strain $\gamma_{x y}$ over the cross-section by Gruttmann et al. (1999) and Gruttmann and Wagner (2001) as well as Kourtis et al. (2009) will be resolved; for two-scale homogenisation, the first order formulation of Karihaloo et al. (2001) will be generalised to higher order following Xiao and Karihaloo (2009).

This paper is organised as follows: we will first discuss the formulation of St Venant torsion of prismatic composite shafts in terms of the warping function and high-performance incompatible, strainenhanced and hybrid stress elements for solving the warping function. This will be followed by the formulation of St Venant bending in terms of the warping function, highlighting its similarity to torsion. We will next discuss the two-scale homogenisation and finite elements for solving the resulting equilibrium equations; followed by numerical examples and finally conclusions and discussion.

\section{St Venant Torsion of Prismatic Composite Shaft}

Take the origin at the centroid of one end section, $z$ axis along the axis of the shaft, $x$ and $y$ axes coincide with the principal axes of the section and form a right hand system with $z$. Orthotropic materials are considered. The principal directions of orthotropy coincide with the coordinate axes. The material properties are uniform along the length, but can be piece-wise constant across the cross-section. The initial stresses and strains will be ignored in the derivation of St Venant solutions, but added in the development of high-performance elements.

Under the assumption that $\sigma_{x}=\sigma_{y}=\sigma_{z}=\tau_{x y}=0$, the displacements corresponding to unit angle of twist per unit length are

$$
\begin{aligned}
& u=-y z \\
& v=x z \\
& w=\varphi(x, y)
\end{aligned}
$$

where $\varphi(x, y)$ is the warping function. The nonvanishing strains are

$$
\begin{aligned}
& \gamma_{x z}=\varphi_{, x}-y \\
& \gamma_{y z}=\varphi_{, y}+x
\end{aligned}
$$

where a comma denotes partial differentiation. The non-vanishing stresses are

$$
\tau_{x z}=G_{x z}\left(\gamma_{x z}-\gamma_{x z}^{0}\right)+\tau_{x z}^{0}
$$




$$
\tau_{y z}=G_{y z}\left(\gamma_{y z}-\gamma_{y z}^{0}\right)+\tau_{y z}^{0}
$$

where $G_{x z}$ and $G_{y z}$ are shear moduli; $\gamma_{x z}^{0}$ and $\gamma_{y z}^{0}$ are initial strains including thermal strains; $\tau_{x z}^{0}$ and $\tau_{y z}^{0}$ are initial stresses.

The equilibrium equation is

$$
\tau_{x z, x}+\tau_{y z, y}=0, \text { in } \mathrm{A}
$$

and boundary condition is

$$
\tau_{x z} n_{x}+\tau_{y z} n_{y}=0 \text {, on } \partial \mathrm{A}
$$

where $\left(n_{x}, n_{y}\right)$ is the unit outward normal to $\partial A$, with A the cross-section domain.

Denote the shear stress vectors

$$
\tau=\left\{\begin{array}{c}
\tau_{x z} \\
\tau_{y z}
\end{array}\right\}, \tau^{0}=\left\{\begin{array}{c}
\tau_{x z}^{0} \\
\tau_{y z}^{0}
\end{array}\right\}
$$

and shear strain vectors

$$
\gamma=\left\{\begin{array}{c}
\gamma_{x z} \\
\gamma_{y z}
\end{array}\right\}=\left\{\begin{array}{c}
\varphi_{, x}-f_{1}(x, y) \\
\varphi_{, y}-f_{2}(x, y)
\end{array}\right\}, \gamma^{0}=\left\{\begin{array}{c}
\gamma_{x z}^{0} \\
\gamma_{y z}^{0}
\end{array}\right\}
$$

where $f_{1}(x, y)=y$ and $f_{2}(x, y)=x$, and modulus and compliance matrices

$$
\mathbf{D}=\left[\begin{array}{cc}
G_{x z} & 0 \\
0 & G_{y z}
\end{array}\right], \mathbf{C}=\left[\begin{array}{cc}
1 / G_{x z} & 0 \\
0 & 1 / G_{y z}
\end{array}\right]
$$

the potential functional whose stationary condition gives (4) and (5) can be written as

$$
\Pi_{p}(\varphi)=\int_{A}\left[\frac{1}{2} \gamma^{2} \mathbf{D} \gamma+\left(-\mathbf{D} \gamma^{0}+\tau^{0}\right)^{T} \gamma\right] d A
$$

The Hellinger-Reissner functional whose stationary condition gives (2)-(5) can be written as

$$
\Pi_{H R}(\varphi, \tau)=\int_{A}\left[-\frac{1}{2} \tau^{T} \mathbf{C} \tau+\tau^{T}\left(\gamma-\gamma^{0}+\mathbf{C} \tau^{0}\right)\right] d A
$$

By relaxing the compatibility condition (2) in the potential functional (9), or employing Legendre transformation on the Hellinger-Reissner functional (10), one arrives at the 3-field $\mathrm{Hu}$-Washizu functional

$$
\begin{aligned}
& \Pi_{H W}(\varphi, \gamma, \tau)= \\
& \int_{A}\left[\frac{1}{2} \gamma^{T} \mathbf{D} \gamma-\tau^{T}\left(\gamma-\left\{\begin{array}{l}
\varphi_{, x}-f_{1} \\
\varphi_{, y}-f_{2}
\end{array}\right\}\right)+\left(-\mathbf{D} \gamma^{0}+\tau^{0}\right)^{T} \gamma\right] d A
\end{aligned}
$$

\section{4-node Incompatible/Strain-enhanced Element}

For the 4-node element shown in Fig. 1, the warping function is divided into a compatible part $\varphi_{q}$ and an incompatible part $\varphi_{\lambda}$ :

$$
\varphi=\varphi_{q}+\varphi_{\lambda}
$$

$\varphi_{q}$ is interpolated from nodal values $\boldsymbol{q}=\left[\varphi_{1}, \varphi_{2} \cdot \varphi_{3}, \varphi_{4}\right]^{\tau}$ using the bilinear shape functions $N_{i}$ as

$$
\varphi_{q}=N \boldsymbol{q}
$$

where

$$
N=\left[N_{1} N_{2} N_{3} N_{4}\right]
$$

and

$$
N_{i}=\frac{1}{4}\left(1+\xi_{i} \xi\right)\left(1+\eta_{i} \eta\right)
$$

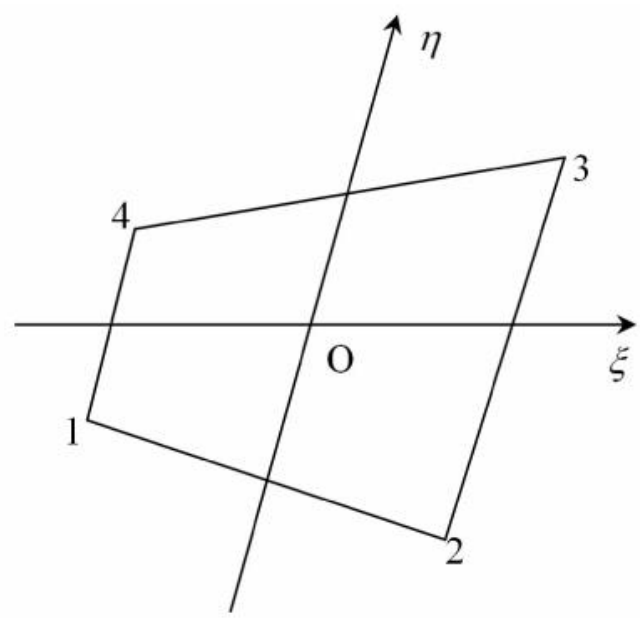

Fig. 1: A plane four-noded quadrilateral element 
$(\xi, \eta)$ represent the element isoparametric coordinates, $\left(\xi_{i}, \eta_{i}\right)$ are the isoparametric coordinates of point $i$ with the global coordinates $\left(x_{i}, y_{i}\right), i=1,2,3,4, \varphi_{\lambda}$ is related to the element inner parameters $\lambda$ via the shape functions $\boldsymbol{N}_{\lambda}$

$$
\varphi_{\lambda}=N_{\lambda} \lambda
$$

Here, two incompatible terms are employed in each element as derived in Karihaloo et al. (2001) and Pian and $\mathrm{Wu}$ (2006)

$$
\begin{aligned}
& N_{\lambda}=\left[N_{\lambda 1} N_{\lambda 2}\right] \\
& N_{\lambda 1}=\xi^{2}-\Delta, N_{\lambda 2}=\eta^{2}+\Delta \\
& \Delta=\frac{2}{3}\left(\frac{J_{1}}{J_{0}} \xi-\frac{J_{2}}{J_{0}} \eta\right)
\end{aligned}
$$

where $J_{0}, J_{1}$ and $J_{2}$ are related to the element Jacobian as follows

$$
\begin{aligned}
& |J|=J_{0}+J_{1} \xi+J_{2} \eta=\left(a_{1} b_{2}-a_{3} b_{1}\right) \\
& +\left(a_{1} b_{2}-a_{2} b_{1}\right) \xi+\left(a_{2} b_{3}-a_{3} b_{2}\right) \eta
\end{aligned}
$$

The coefficients and $(i=1,2,3)$ are dependent on the element nodal co-ordinates $\left(x_{i}, y_{i}\right)(i=1,2,3$, 4) as follows:

$$
\left|\begin{array}{ll}
a_{1} & b_{1} \\
a_{2} & b_{2} \\
a_{3} & b_{3}
\end{array}\right|=\frac{1}{4}\left|\begin{array}{cccc}
-1 & 1 & 1 & -1 \\
1 & -1 & 1 & -1 \\
-1 & -1 & 1 & 1
\end{array}\right|\left|\begin{array}{ll}
x_{1} & y_{1} \\
x_{2} & y_{2} \\
x_{3} & y_{3} \\
x_{4} & y_{4}
\end{array}\right|
$$

With the above assumed warping function (12) together with (13) and (16), we have the shear strains from (7)

$$
\begin{aligned}
\boldsymbol{\gamma}=\left\{\begin{array}{l}
\gamma_{x z} \\
\gamma_{y z}
\end{array}\right\} & =\left\{\begin{array}{l}
\frac{\partial}{\partial x} \\
\frac{\partial}{\partial y}
\end{array}\right\}\left[\begin{array}{ll}
\boldsymbol{N} & \boldsymbol{N}_{\lambda}
\end{array}\right]\left\{\begin{array}{l}
\boldsymbol{q} \\
\boldsymbol{\lambda}
\end{array}\right\}+\left\{\begin{array}{l}
-f_{1} \\
f_{2}
\end{array}\right\} \\
& =\left[\begin{array}{ll}
\mathbf{B} & \mathbf{B}_{\lambda}
\end{array}\right]\left\{\begin{array}{l}
\boldsymbol{q} \\
\boldsymbol{\lambda}
\end{array}\right\}+\left\{\begin{array}{l}
-f_{1} \\
f_{2}
\end{array}\right\}
\end{aligned}
$$

Substituting (20) into (9) and making use of the stationary condition, yields

$$
\tilde{\mathbf{K}} \boldsymbol{q}=\boldsymbol{f}
$$

where the element stiffness matrix and nodal vector are

$$
\tilde{\mathbf{K}}=\mathbf{K}_{q q}-\mathbf{K}_{q \lambda} \mathbf{K}_{\lambda \lambda}^{-1} \mathbf{K}_{q \lambda}^{T}, \boldsymbol{f}=\mathbf{K}_{q \lambda} \mathbf{K}_{\lambda \lambda}^{-1} \boldsymbol{f}_{\lambda}-\boldsymbol{f}_{q}
$$

in which

$$
\begin{aligned}
& {\left[\begin{array}{ll}
\mathbf{K}_{q q} & \mathbf{K}_{q \lambda} \\
\mathbf{K}_{q \lambda}^{T} & \mathbf{K}_{\lambda \lambda}
\end{array}\right]=\int_{A}\left[\begin{array}{l}
\mathbf{B}^{T} \\
\mathbf{B}_{\lambda}^{T}
\end{array}\right] \mathbf{D}\left[\begin{array}{ll}
\mathbf{B} & \mathbf{B}_{\lambda}
\end{array}\right] d A} \\
& \left\{\begin{array}{l}
f_{q} \\
f_{\lambda}
\end{array}\right\}=\int_{A}\left[\begin{array}{l}
\mathbf{B}^{T} \\
\mathbf{B}_{\lambda}^{T}
\end{array}\right]\left[\mathbf{D}\left(\left\{\begin{array}{c}
-f_{1} \\
f_{2}
\end{array}\right\}-\gamma^{0}\right)+\tau^{0}\right] d A
\end{aligned}
$$

The element inner parameters $\lambda$ are recovered as follows

$$
\lambda=-\mathbf{K}_{\lambda \lambda}^{-1} \mathbf{K}_{q \lambda}^{T} \boldsymbol{q}-\mathbf{K}_{\lambda \lambda}^{-1} \boldsymbol{f}_{\lambda}
$$

Alternatively, we can substitute the compatible bilinear interpolation of warping function in (13) and the shear strains (20) (the shear strains compatible with warping (13) are enhanced with $\mathbf{B}_{\lambda} \boldsymbol{\lambda}$ ) into the $\mathrm{Hu}$-Washizu potential (11), an equivalent strainenhanced element can be obtained.

\section{4-node Hybrid Stress Element}

In optimisation of the element trial stresses, the constant stresses are generally isolated to remain unperturbed to ensure the element is able to pass patch tests, see e.g., Pian and Wu (2005). Since St Venant torsion cannot have a constant shear stress field, in the development of the 4-node hybrid stress element, Xiao et al. (1999) did not isolate the constant shear stresses in optimisation of the element trial stresses and obtained the following optimised trial stresses

$$
\left\{\begin{array}{c}
\tau_{x z} \\
\tau_{y z}
\end{array}\right\}=\left[\begin{array}{cccc}
a_{1} b_{3}-a_{1} b_{2} \xi & a_{1} a_{2} \xi & a_{1} \eta & a_{3} \xi \\
b_{2} b_{3} \eta & a_{1} b_{3}-a_{2} b_{3} \eta & b_{1} \eta & b_{3} \xi
\end{array}\right]\left[\begin{array}{c}
\beta_{1} \\
\beta_{2} \\
\beta_{3} \\
\beta_{4}
\end{array}\right\}
$$

with 4 stress parameters $\beta_{1}, \beta_{2}, \beta_{3}$ and $\beta_{4}$. The trial stresses (25) satisfy the optimal parameter matching condition, and the resulting element performs as good 
as the well-known Pian-Sumihara element for plane stress/strain problems (Pian and Sumihara, 1984).

However, the element with trial stresses (25) will fail for a square element with nodes numbered as in Fig. 2(a), which has nodal coordinates

$$
\begin{aligned}
& x_{1}=x_{2}=-a, x_{3}=x_{4}=a,=y_{1}=y_{4}=a \\
& \text { and } y_{2}=y_{3}=-a
\end{aligned}
$$

giving

$$
a_{1}=a_{2}=0, a_{3}=a \text { and } b_{1}=-a, b_{2}=b_{3}=0
$$

with the use of (19). The trial stresses (25) become

$$
\left\{\begin{array}{c}
\tau_{x z} \\
\tau_{y z}
\end{array}\right\}=\left[\begin{array}{cccc}
0 & 0 & 0 & a \xi \\
0 & 0 & -a \eta & 0
\end{array}\right]\left[\begin{array}{c}
\beta_{1} \\
\beta_{2} \\
\beta_{3} \\
\beta_{4}
\end{array}\right\}
$$

and the resulting $\mathbf{H}$ matrix (its definition is given in (37) below) will not be invertible. This problem can be resolved by renumbering the nodes into the pattern shown in Fig. 2(b), which gives

(A)

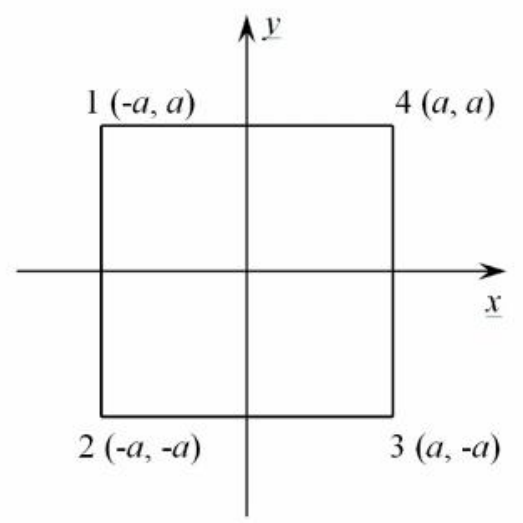

(B)

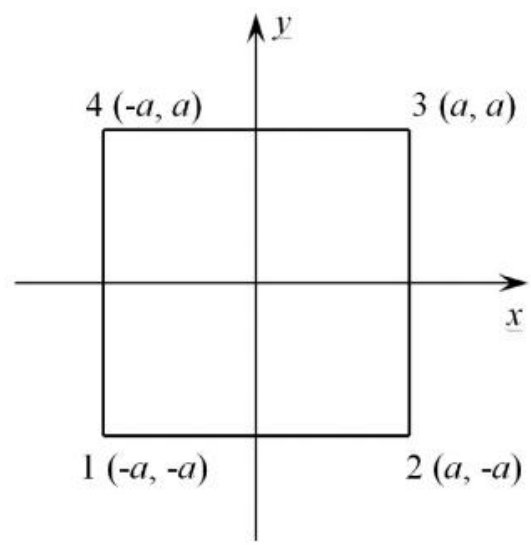

Fig. 2: Two typical node numbering patterns for a 4-node square element with side length $2 a$ $x_{1}=x_{4}=-a, x_{2}=x_{3}=a,=y_{2}=-a y_{4}=a$

and $y_{3}=y_{4}=a$

and

$a_{1}=a, a_{2}=a_{3}=0, b_{1}=b_{2}=0, b_{3}=a$

The trial stresses (25) become

$$
\left\{\begin{array}{c}
\tau_{x z} \\
\tau_{y z}
\end{array}\right\}=\left[\begin{array}{cccc}
a^{2} & 0 & a \eta & 0 \\
0 & a^{2} & 0 & a \xi
\end{array}\right]\left[\begin{array}{l}
\beta_{1} \\
\beta_{2} \\
\beta_{3} \\
\beta_{4}
\end{array}\right\}
$$

and the resulting $\mathbf{H}$ matrix will be invertible.

Alternatively, the constant shear stresses can be isolated from the stress optimisation procedure, and the initial assumed stresses of Xiao et al. (1999) can now be divided into constant and higher order parts as

$$
\begin{aligned}
& \left\{\begin{array}{c}
\tau_{x z} \\
\tau_{y z}
\end{array}\right\}=\left[\begin{array}{llllll}
1 & 0 & \eta & 0 & \xi & 0 \\
0 & 1 & 0 & \xi & 0 & \eta
\end{array}\right]\left\{\begin{array}{c}
\beta_{1} \\
\vdots \\
\beta_{6}
\end{array}\right\} \\
& =\beta_{c}+\left[\varphi_{I} \mid \varphi_{I I}\right]\left\{\begin{array}{l}
\beta_{I} \\
\beta_{I I}
\end{array}\right\} \\
& \beta_{c}=\left\{\begin{array}{l}
\beta_{1} \\
\beta_{2}
\end{array}\right\}, \beta_{I}=\left\{\begin{array}{c}
\beta_{3} \\
\beta_{4}
\end{array}\right\}, \beta_{I I}=\left\{\begin{array}{c}
\beta_{5} \\
\beta_{6}
\end{array}\right\} \text {, } \\
& \varphi_{I}=\left[\begin{array}{ll}
\eta & 0 \\
0 & \xi
\end{array}\right], \varphi_{I I}=\left[\begin{array}{ll}
\xi & 0 \\
0 & \eta
\end{array}\right]
\end{aligned}
$$

Enforcement of the stress optimisation condition on the higher order stresses $\left(\varphi_{I} \beta_{I}+\varphi_{I I} \beta_{I I}\right)$ in (32) following Xiao et al. (1999) (or Pian and Wu (2005)) results in

$$
\beta_{I I}=\left[\begin{array}{cc}
0 & \frac{a_{3}}{b_{3}} \\
\frac{b_{1}}{a_{1}} & 0
\end{array}\right] \beta_{I}
$$

Substitution of (33) into (32) gives the final optimised trial stresses

$$
\left\{\begin{array}{c}
\tau_{x z} \\
\tau_{y z}
\end{array}\right\}=\left[\begin{array}{llll}
1 & 0 & a_{1} \eta & a_{3} \xi \\
0 & 1 & b_{1} \eta & b_{3} \xi
\end{array}\right]\left\{\begin{array}{c}
\beta_{1} \\
\beta_{2} \\
\beta_{3} \\
\beta_{4}
\end{array}\right\} \text {, or } \tau=\phi \beta
$$


Substitution of the warping function (13) into (7) gives the shear strain vector

$$
\boldsymbol{\gamma}=\mathbf{J}^{-1}\left[\begin{array}{l}
\boldsymbol{N}_{, \xi} \\
\boldsymbol{N}_{, \eta}
\end{array}\right] \boldsymbol{q}+\left\{\begin{array}{l}
-y \\
x
\end{array}\right\}=\mathbf{B} \boldsymbol{q}+\left\{\begin{array}{l}
-y \\
x
\end{array}\right\}
$$

Substitution of (34) and (35) into (10) gives

$$
\Pi_{H P}(\boldsymbol{q}, \beta)=-\frac{1}{2} \beta^{T} \boldsymbol{H} \beta+\beta^{T}(\mathbf{G} \boldsymbol{q}+\hat{\boldsymbol{f}})
$$

where

$$
\begin{aligned}
\mathbf{H} & =\int_{A} \phi^{T} \mathbf{C} \phi d A \\
\mathbf{G} & =\int_{A} \phi^{T} \boldsymbol{B} d A \\
\widehat{\boldsymbol{f}} & =\int_{A} \phi^{T}\left(\left\{\begin{array}{l}
-\boldsymbol{f}_{\boldsymbol{1}} \\
f_{2}
\end{array}\right\}-\gamma^{0}+\mathbf{C} \boldsymbol{\tau}^{0}\right) d A
\end{aligned}
$$

Vanishing of the first order variation of $\Pi_{H R}$ (36) gives

$$
-\mathbf{H} \beta+\mathbf{G} \boldsymbol{q}+\hat{\boldsymbol{f}}=0, \beta^{T} \mathbf{G}=0
$$

The stress parameters can be computed from the first equation of (38)

$$
\beta=\mathbf{H}^{-1} \mathbf{G} \boldsymbol{q}+\mathbf{H}^{-1} \hat{\boldsymbol{f}}
$$

Substitution of (39) into the second equation of (38) gives

$$
\mathbf{G}^{T} \mathbf{H}^{-1} \mathbf{G} \boldsymbol{q}=-\mathbf{G}^{\boldsymbol{T}} \mathbf{H}^{-1} \hat{\boldsymbol{f}}
$$

or the stiffness matrix

$$
\mathbf{K}=\mathbf{G}^{T} \mathbf{H}^{-1} \mathbf{G}
$$

and nodal load vector

$$
\boldsymbol{f}=-\mathbf{G}^{T} \mathbf{H}^{-1} \hat{\boldsymbol{f}}
$$

of the element.

Numerical tests confirm that the element resulting from (34) performs as good as the original element using trial stresses (25), but does not require renumbering the nodes for the special pattern shown in Fig. 2(a).

\section{St Venant Torsionless Bending of Prismatic Composite Shaft}

Under the assumption that the normal strain $\varepsilon_{z}$ is linear with respect to $x, y$ and $z$, according to the elementary beam theory, and stresses $\sigma_{x}, \sigma_{y}$ and $\tau_{x y}$ are neglected

$$
\begin{aligned}
& \varepsilon_{z}=\left(\hat{a}_{1}+\hat{a}_{2} z\right) x+\left(\hat{b}_{1}+\hat{b}_{2} z\right) y \\
& \sigma_{x}=\sigma_{y}=\tau_{x y}=0
\end{aligned}
$$

where $\hat{a}_{1}, \hat{a}_{2}, \hat{b}_{1}$ and $\hat{b}_{2}$ are constants, the non-vanishing strains are related to the stresses as

$$
\begin{aligned}
& \varepsilon_{x}=-\frac{v_{z x}}{E_{z}} \sigma_{z} \\
& \varepsilon_{y}=-\frac{v_{z y}}{E_{z}} \sigma_{z} \\
& \varepsilon_{x}=-\frac{v_{z x}}{E_{z}} \sigma_{z} \\
& \varepsilon_{z}=\frac{1}{E_{z}} \sigma_{z} \\
& \gamma_{y z}=\frac{1}{G_{y z}} \tau_{y z} \\
& \gamma_{x z}=\frac{1}{G_{x z}} \tau_{x z}
\end{aligned}
$$

The displacements

$$
\left.u=-v_{z x}\left[\frac{1}{2}\left(a_{1}+\hat{a}_{2} z\right) x^{2}+\left(\hat{b}_{1}+\hat{b}_{2} z\right) x y\right)\right]
$$

$$
+g_{1}(y, z)
$$

$$
\begin{gathered}
\left.v=-v_{z y}\left[\left(\hat{a}_{1}+\hat{a}_{2} z\right) x y+\frac{1}{2}\left(\hat{b}_{1}+\hat{b}_{2} z\right) y^{2}\right)\right] \\
+g_{2}(x, z)
\end{gathered}
$$

$$
\begin{aligned}
w= & \left(\hat{a}_{1}+\hat{a}_{2} z+\frac{1}{2} \hat{a}_{2} z^{2}\right) x \\
& +\left(\hat{b}_{0}+\hat{b}_{1} z+\frac{1}{2} \hat{b}_{2} z^{2}\right) y+\varphi(x, y)
\end{aligned}
$$


where $\hat{a}_{0}$ and $\hat{b}_{0}$ are constants; $\varphi(x, y)$ is the warping function due to bending, and

$$
\begin{aligned}
& g_{1}(y, z)=\frac{1}{2} v_{z y}\left(\hat{a}_{1}+\hat{a}_{2} z\right) y^{2}-h_{1}(z) \\
& g_{2}(x, z)=\frac{1}{2} v_{z x}\left(\hat{b}_{1}+\hat{b}_{2} z\right) x^{2}-h_{2}(z)
\end{aligned}
$$

and functions $h_{1}(z)$ and $h_{1}(z)$ satisfy

$$
\begin{aligned}
& h_{1, z}=\hat{a}_{0}+\hat{a}_{1} z+\frac{1}{2} \hat{a}_{2} z^{2} \\
& h_{2, z}=\hat{b}_{0}+\hat{b}_{1} z+\frac{1}{2} b_{2} z^{2}
\end{aligned}
$$

The non-vanishing shear strains are

$$
\begin{aligned}
& \gamma_{x z}=\varphi_{, x}-f_{1}(x, y) \\
& \gamma_{y z}=\varphi_{, y}-f_{2}(x, y)
\end{aligned}
$$

where

$$
\begin{aligned}
& f_{1}(x, y)=v_{z x}\left(\frac{1}{2} \hat{a}_{2} x^{2}+\hat{b}_{2} x y\right)-\frac{1}{2} v_{z y} \hat{a}_{2} y^{2} \\
& f_{2}(x, y)=-v_{z y}\left(\hat{a}_{2} x y+\frac{1}{2} \hat{b}_{2} y^{2}\right)+\frac{1}{2} v_{z x} \hat{b}_{2} x^{2}
\end{aligned}
$$

The shear stresses are related to the shear strains (48) in the same manner as (3) after adding the initial stresses and strains into (44) and satisfying the equilibrium equation

$$
\tau_{x z, x}+\tau_{y z, y}=-\sigma_{z, z}=-E_{z}\left(\hat{a}_{2} x+\hat{b}_{2} y\right)=f_{0}(x, y), \text { in }
$$

A

and boundary condition for stress free cylindrical surface (5).

Constants $\hat{a}_{2}$ and $\hat{b}_{2}$ must be known before solving for the warping function due to bending; they can be computed from the shear forces $Q_{x}$ and $Q_{y}$ as follows.

$$
\begin{aligned}
& \hat{a}_{2}=\frac{E I_{x x} Q_{x}-E I_{x y} Q_{y}}{E I_{y y} \cdot E I_{x x}-\left(E I_{x y}\right)^{2}}, \\
& \hat{b}_{2}=\frac{E I_{y y} Q_{y}-E I_{x y} Q_{x}}{E I_{y y} \cdot E I_{x x}-\left(E I_{x y}\right)^{2}}
\end{aligned}
$$

where

$$
\begin{aligned}
E I_{x x} & =\int_{A} E_{z} y^{2} d A, E I_{y y}=\int_{A} E_{z} x^{2} d A, E I_{x y} \\
& =\int_{A} E_{z} x y d A
\end{aligned}
$$

A comparison with the formulation of St Venant torsion discussed above shows that the present formulation for St Venant bending appears identical to torsion except that a body force $f_{0}(x, y)$ now appears. The 4-node quadrilateral incompatible/strainenhanced and hybrid stress elements for torsion can thus be readily used for the analysis of St Venant bending.

The potential functional whose stationary condition gives (50) and (5) now becomes

$$
\begin{aligned}
& \Pi_{p}(\varphi)= \\
& \int_{A}\left[\frac{1}{2} \gamma^{T} \mathbf{D} \gamma+\left(-\mathbf{D} \gamma^{0}+\tau^{0}\right)^{T} \gamma+f_{0} \varphi\right] d A
\end{aligned}
$$

The Hellinger-Reissner functional whose stationary condition gives (48) together with (3), (50) and boundary condition (5) becomes

$$
\begin{aligned}
& \Pi_{H R}(\varphi, \tau)= \\
& \int_{A}\left[-\frac{1}{2} \tau^{T} \mathbf{C} \tau+\tau^{\tau}\left(\gamma-\gamma^{0}+\mathbf{C} \tau^{0}\right)+f_{0} \varphi\right] d A
\end{aligned}
$$

By relaxing the compatibility condition (48) in the potential functional (53), or employing Lengendre transformation on the Hellinger-Reissner functional (54), one arrives at the $\mathrm{Hu}$-Washizu functional

$$
\begin{aligned}
& \Pi_{H w}(\varphi, \gamma, \tau)= \\
& \int_{A}\left[\frac{1}{2} \boldsymbol{\tau}^{T} \mathbf{D} \gamma-\tau^{\tau}\left(\gamma-\left\{\begin{array}{ll}
\varphi_{, x} & -f_{1}(x, y) \\
\varphi_{, y} & +f_{2}(x, y)
\end{array}\right\}\right)\right. \\
& \left.+\left(-D \gamma^{0}+\tau^{0}\right)^{T} \gamma+f_{0} \varphi\right] d A
\end{aligned}
$$

\section{Incompatible/Strain-enhanced Element}

Substitution of (12) together with (13) and (16) into (48) gives the shear strains (20). Substitution of (20) into (53) yields (21)-(24) with the only difference that 


$$
\begin{aligned}
& \left\{\begin{array}{l}
f_{q} \\
f_{\lambda}
\end{array}\right\}=\int_{A}\left[\begin{array}{l}
\mathbf{B}^{T} \\
\mathbf{B}_{\lambda}^{T}
\end{array}\right] \\
& {\left[\mathbf{D}\left(\left\{\begin{array}{c}
-f_{1} \\
f_{2}
\end{array}\right\}-\gamma^{0}\right)+\tau^{0}\right] d A+\int_{A}\left\{\begin{array}{l}
\boldsymbol{N}^{T} \\
\boldsymbol{N}_{\lambda}^{T}
\end{array}\right\} f_{0} d A}
\end{aligned}
$$

in place of (23).

\section{Hybrid Stress Element}

Substitution of the warping and shear stress interpolations (13) and (34) into (54) yields

$$
\begin{aligned}
& \Pi_{H R}(\boldsymbol{q}, \boldsymbol{\beta})=-\frac{1}{2} \beta^{T} \mathbf{H} \boldsymbol{\beta} \\
& +\beta^{T}(\mathbf{G} \boldsymbol{q}+\hat{\boldsymbol{f}})+\hat{\boldsymbol{f}}^{T} \boldsymbol{q}
\end{aligned}
$$

where $\mathbf{H}, \mathbf{G}$ and $\hat{\boldsymbol{f}}$ are the same as (37), but

$$
\hat{\boldsymbol{f}}=\int_{A} \boldsymbol{N}^{T} f_{0} d A
$$

Vanishing of the first order variation of $\Pi_{H R}$ (57) gives

$$
\begin{aligned}
& -\mathbf{H} \beta+\mathbf{G} \boldsymbol{q}+\hat{\boldsymbol{f}}=0 \\
& \beta^{T} \mathbf{G}+\hat{\boldsymbol{f}}^{T}=0
\end{aligned}
$$

From the first equation of (59) we can obtain the stress parameters in the same manner as (39), substitution of them into the second equation of (59) gives

$$
\mathbf{G}^{T} \mathbf{H}^{-1} \mathbf{G} \boldsymbol{q}=-\mathbf{G}^{T} \mathbf{H}^{-1} \hat{\boldsymbol{f}}-\hat{\boldsymbol{f}}
$$

The stiffness matrix is the same as (41), but the nodal load vector now becomes

$$
\boldsymbol{f}=-\mathbf{G}^{T} \mathbf{H}^{-1} \hat{\boldsymbol{f}}-\hat{\boldsymbol{f}}
$$

\section{Two-scale Asymptotic Homogenisation}

Assume the microstructure of the cross-section $A^{\varepsilon}$ occupied by the composite material to be locally periodic with a period defined by a statistically homogeneous volume element, denoted by the representative unit cell (RUC) or volume element (RVE) with size $Y$, as shown in Fig. 3. In other words, the composite material is formed by a spatial repetition

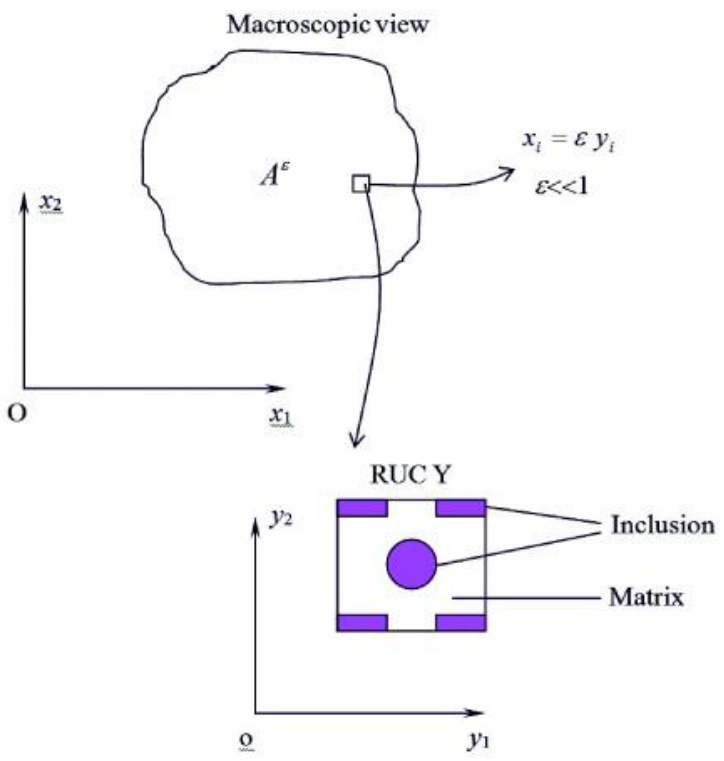

Fig. 3: Illustration of a problem with two length scales

of the RUC. The problem has two length scales; a global length scale $D$ that is of the order of the size of section $A^{\varepsilon}$, and a local length scale $d$ that is of the order of the RUC and proportional to the wavelength of the variation of the micro-structure. The size of the RUC is much larger than that of the constituents but much smaller than that of the section. The relation between the global coordinate system $x_{i}$ for the section and the local system $y_{i}$ for the minimum RUC can then be written as

$$
y_{i}=\frac{x_{i}}{\varepsilon} i=1,2
$$

where $\varepsilon$ is a very small positive number representing the scaling factor between the two length scales. The local coordinate vector $y_{i}$ is regarded as a stretched coordinate vector in the microscopic domain.

For an actual composite shaft subjected to external forces, field quantities such as warping function, shear strains and stresses are assumed to have slow variations from point to point with macroscopic (global) coordinate $x$ as well as fast variations with local microscopic coordinate $y$ within a small neighbourhood of size $\varepsilon$ of a given point $x$

$$
\begin{aligned}
& u_{3}^{\varepsilon}=u_{3}^{\varepsilon}(x, y)=\varphi^{\varepsilon}(x, y) \\
& \gamma_{3 j}^{\varepsilon}=\gamma_{3 j}^{\varepsilon}(x, y) \\
& \tau_{3 j}^{\varepsilon}=\tau_{3 j}^{\varepsilon}(x, y)
\end{aligned}
$$


where $j=1,2$. The superscript $\varepsilon$ denotes Y-periodicity of the corresponding function.

The unknown displacement $u_{3}^{\varepsilon}$, the non-zero strain $\gamma_{3 j}^{\varepsilon}$ and stress $\tau_{3 j}^{\varepsilon}$ can be solved from the following equations

Equilibrium:

$$
\frac{\partial \tau_{3 j}^{\varepsilon}}{\partial x_{j}}=f_{0} \text { in } A^{\varepsilon}
$$

where $f_{0}=0$ for torsion; it is defined as in (50) for bending.

Kinematical:

$$
\left\{\begin{array}{l}
\gamma_{31}^{\varepsilon} \\
\gamma_{32}^{\varepsilon}
\end{array}\right\}=\left\{\begin{array}{ll}
\frac{\partial u_{3}^{\varepsilon}}{\partial x_{1}} & f_{1}(x, y) \\
\frac{\partial u_{3}^{\varepsilon}}{\partial x_{2}} & f_{2}(x, y)
\end{array}\right\} \text { in } A^{\varepsilon}
$$

Constitutive:

$$
\tau_{3 i}^{\varepsilon}=D_{i j}^{\varepsilon}\left(\gamma_{3 j}^{\varepsilon}-\gamma_{3 j}^{0}\right)+\tau_{3 j}^{0} \quad \text { in } \quad A^{\varepsilon}
$$

together with the traction free condition on the surface of the shaft, and the traction and displacement conditions at the interfaces between the microconstituents. The material property tensor $D_{i j}^{\varepsilon}$ is symmetric with respect to indices $(i, j)$. The convention of summation over the repeated indices is used.

The displacement $u_{3}^{\varepsilon}(x, y)$ is expanded in powers of the small number $e$ as

$$
\begin{aligned}
& u_{3}^{\varepsilon}(x, y)=u_{3}^{(0)}(x, y)+\varepsilon u_{3}^{(1)}(x, y) \\
& +\varepsilon^{2} u_{3}^{(2)}(x, y)+\varepsilon^{3} u_{3}^{(3)}(x, y) \ldots
\end{aligned}
$$

where $u_{3}^{(0)}, u_{3}^{(1)}, u_{3}^{(2)}, \ldots$, are $Y$-periodic functions with respect to $y$. Substituting (67) into (65) gives the expansion of the strain $\gamma_{3 j}^{\varepsilon}$ :

$$
\gamma_{3 j}^{\varepsilon}(x, y)=\varepsilon^{-1} \gamma_{3 j}^{(-1)}+\gamma_{3 j}^{(0)}+\varepsilon \gamma_{3 j}^{(-1)}+\ldots .
$$

where

$$
\begin{aligned}
& \gamma_{3 j}^{(-1)}=\frac{\partial u_{3}^{(0)}}{\partial y_{j}} \\
& \gamma_{3 j}^{(0)}=\gamma_{x 3 j}^{(0)}+\gamma_{y 3 j}^{(0)}, \gamma_{x 3 j}^{(0)}=\frac{\partial u_{3}^{(0)}}{\partial x_{1}}-f_{1} . \\
& \gamma_{x 32}^{(0)}=\frac{\partial u_{3}^{(0)}}{\partial x_{1}}+f_{2}, \gamma_{y 3 j}^{(0)}=\frac{\partial u_{3}^{(1)}}{\partial y_{j}} \\
& \gamma_{3 j}^{(k)}=\frac{\partial u_{3}^{(k)}}{\partial x_{j}}+\frac{\partial u_{3}^{(k+1)}}{\partial y_{j}}, k \geq 1
\end{aligned}
$$

Substituting (68) into the constitutive relation (66) gives the expansion of the stress $\tau_{3 j}^{\varepsilon}$

$$
\tau_{3 j}^{\varepsilon}(x, y)=\varepsilon^{-1} \tau_{3 j}^{(-1)}+\tau_{3 j}^{(0)}+\varepsilon \tau_{3 j}^{(1)}+\ldots
$$

where

$$
\begin{aligned}
& \tau_{3 i}^{(-1)}=D_{i j} \gamma_{3 j}^{(-1)} \\
& \tau_{3 i}^{(0)}=D_{i j}\left(\gamma_{3 j}^{(0)}-\gamma_{3 j}^{(0)}\right)+\tau_{3 j}^{0} \\
& \tau_{3 i}^{(k)}=D_{i j} \gamma_{3 j}^{(k)}, k \geq 1
\end{aligned}
$$

Inserting the asymptotic expansion for the stress field (70) into the equilibrium equation (64) and collecting the terms of like powers in $\varepsilon$ gives the following sequence of equilibrium equations

$$
O\left(\varepsilon^{-2}\right): \quad \frac{\partial \tau_{3 j}^{(-1)}}{\partial y_{j}}=0
$$

$$
O\left(\varepsilon^{-1}\right): \quad \frac{\partial \tau_{3 j}^{(-1)}}{\partial x_{j}}+\frac{\partial \tau_{3 j}^{(0)}}{\partial y_{j}}=0
$$

$O\left(\varepsilon^{0}\right): \quad \frac{\partial \tau_{3 j}^{(0)}}{\partial x_{j}}+\frac{\partial \tau_{3 j}^{(1)}}{\partial y_{j}}=f_{0}$

$$
O\left(\varepsilon^{k}\right): \quad \frac{\partial \tau_{3 j}^{(k)}}{\partial x_{j}}+\frac{\partial \tau_{3 j}^{(k+1)}}{\partial y_{j}}=0, k \geq 1
$$


$O\left(\varepsilon^{-2}\right)$ Equilibrium: Solution Structure of $u_{3}^{(0)}$

We first consider the $\mathrm{O}\left(\varepsilon^{-2}\right)$ equilibrium equation (72) in Y. Pre-multiplying it by $u_{3}^{(0)}$, integrating over $\mathrm{Y}$, followed by integration by parts, yields

$$
\begin{aligned}
& \int_{Y} u_{3}^{(0)} \frac{\partial \tau_{3 j}^{(-1)}}{\partial y_{j}} d Y=\oint_{\partial Y} u_{3}^{(0)} \tau_{3 j}^{(-1)} n_{j} d \Gamma- \\
& \int_{Y} \frac{\partial u_{3}^{(0)}}{\partial y_{j}} D_{j i} \frac{\partial u_{3}^{(0)}}{\partial y_{i}} d Y=0
\end{aligned}
$$

where $\partial Y$ denotes the boundary of $Y$. The boundary integral term in (76) vanishes due to the periodicity of the boundary conditions in $Y$, because $u_{3}^{(0)}$ and $\tau_{3 j}^{(-1)}$ are identical on the opposite sides of the unit cell, while the corresponding normals $d_{j}$ are in opposite directions. Taking into account the positive definiteness of the symmetric constitutive tensor $D_{i j}$, we have

$$
\frac{\partial u_{3}^{(0)}}{\partial y_{j}}=0 \Rightarrow u_{3}^{(0)}=u_{3}^{(0)}(x)
$$

and

$$
\gamma_{3 j}^{(-1)}(x, y)=0, \quad \tau_{3 j}^{(-1)}(x, y)=0
$$

\section{$O\left(\varepsilon^{-1}\right)$ Equilibrium: First-Order Homogeni-} sation and Solution Structure of $\boldsymbol{u}_{3}^{(1)}$

Next, we proceed to the $O\left(\varepsilon^{01}\right)$ equilibrium equation (73). From (69) and (71) and taking into account (78), it follows that

$$
\frac{\partial \tau_{3 j}^{(0)}}{\partial y_{j}}=0
$$

or

$$
\frac{\partial}{\partial y_{j}}\left(D_{j i} \gamma_{y 3 i}^{(0)}\left(u_{3}^{(1)}\right)\right)=-\frac{\partial D_{j i}}{\partial y_{j}}\left[\gamma_{x 3 i}^{(0)}\left(u_{3}^{(0)}\right)-\gamma_{3 i}^{0}\right]
$$

assuming that

$$
\gamma_{3 i}^{0}=\gamma_{3 i}^{0}(x), \tau_{3 i}^{0}=\tau_{3 i}^{0}(x)
$$

Based on the form of the right-hand side of (80) which permits a separation of variables, $u_{3}^{(1)}$ may be expressed as

$$
u_{3}^{(1)}(x, y)=\chi_{3}^{3 j}(v)\left|\gamma_{x 3 j}^{(0)}\left(u_{3}^{(0)}\right)-\gamma_{3 j}^{(0)}\right|
$$

where $\chi_{3}^{3 j}(y)$ is a $Y$-periodic function defined in the unit cell $Y$. Substituting (82) into (80), and taking into account the arbitrariness of the macroscopic strain field, $\gamma_{x 3 j}\left(u_{3}^{(0)}\right)-\gamma_{3 j}^{0}$ within a unit cell, we have

$$
\frac{\partial}{\partial y_{j}}\left(D_{j i} \gamma_{y 3 i}^{(0)}\left(\chi_{3}^{3 k}(y)\right)\right)=-\frac{\partial D_{j k}}{\partial y_{j}}
$$

We can also write

$$
\tau_{3 j}^{(0)}=D_{j l}\left(\delta_{\ln }+\frac{\partial \chi_{3}^{3 n}}{\partial y_{l}}\right)\left(\gamma_{x 3 n}^{(0)}-\gamma_{3 n}^{0}\right)+\tau_{3 j}^{0}
$$

\section{$O\left(\varepsilon^{0}\right)$ Equilibrium: Second-Order Homogeni-} sation

We now consider the $O\left(\varepsilon^{0}\right)$ equilibrium equation (74).

\section{Solution Structure of $u_{3}^{(2)}$}

Without loss of generality, assume $\partial f_{0} / \partial f_{i}=0$. Differentiating equation (74) with respect to $y_{i}$ gives

$$
\frac{\partial^{2} \tau_{3 j}^{(0)}}{\partial y_{i} \partial x_{j}}+\frac{\partial^{2} \tau_{3 j}^{(1)}}{\partial y_{i} \partial y_{j}}=\frac{\partial f_{0}}{\partial y_{i}}=0
$$

From (69) and (71), and making use of (82)

$$
\begin{aligned}
& \tau_{3 i}^{(i)}=D_{i j} \gamma_{3 j}^{(1)}=D_{i j}\left(\frac{\partial u_{3}^{(1)}}{\partial x_{j}}+\frac{\partial u_{3}^{(2)}}{\partial y_{j}}\right) \\
& =D_{i j}\left[\chi_{3}^{3 n}(\mathbf{y}) \frac{\partial}{\partial x_{j}}\left(\gamma_{x 3 n}^{(0)}\left(u_{3}^{(0)}\right)-\gamma_{3 n}^{0}(\mathbf{x})\right)+\frac{\partial u_{3}^{(2)}}{\partial y_{j}}\right]
\end{aligned}
$$


Making use of (84) and (86), we thus have

$$
u_{3}^{(2)}(\mathbf{x}, \mathbf{y})=\psi_{3}^{3 n o}(\mathbf{y}) \frac{\partial}{\partial x_{o}}\left(\gamma_{x 3 n}^{(0)}\left(u_{3}^{(0)}\right)-\gamma_{3 n}^{0}(\mathbf{x})\right.
$$

from (85).

\section{Solution of $u_{3}^{0}$}

Integrating (74) over the unit cell domain $Y$ yields

$$
\frac{\partial}{\partial x_{j}} \int_{Y} \tau_{3 j}^{(0)} d Y+\int_{Y} \frac{\partial \tau_{3 j}^{(1)}}{\partial y_{j}} d Y=\int_{Y} f_{0} d Y
$$

Taking into account the periodicity of $\tau_{3 j}^{(1)}$ on $\mathrm{Y}$, the second term vanishes. Substituting (84) into (88) yields

$$
\frac{\partial}{\partial x_{j}}\left\{\bar{D}_{j l}\left[\gamma_{x 3 l}^{(0)}\left(u_{3}^{(0)}\right)-\gamma_{3 l}^{0}\right]+\tau_{3 j}^{0}\right\}=f_{0}
$$

This is an equilibrium equation for a homogeneous medium (cf. (64)) with constant material properties $\bar{D}_{j l}$, which are usually termed as the homogenised or effective material properties and are given by

$$
\bar{D}_{j n}=\frac{1}{Y} \int_{Y} \tilde{D}_{j n} d Y, \tilde{D}_{j n}=\tilde{D}_{j l}\left(\delta_{l n}+\frac{\partial \chi_{3}^{3 n}}{\partial y_{l}}\right)
$$

where $Y$ is the area of the unit cell.

In the widely used first-order homogenisation, displacements to order are solved; in a like manner the equations to order $O\left(\varepsilon^{-1}\right)$ are considered. Equation (89) results from constraints from higherorder equilibrium and is used directly to solve for $u_{3}^{(0)}$. Hence no more constraints are required.

\section{Solution of $\psi_{3}^{3 n o}(y)$}

With the use of (84), (86), (87) and (90), (74) becomes

$$
\frac{\partial}{\partial y_{j}}\left[D_{j l} \frac{\partial \Psi_{3}^{3 n o}(\mathbf{y})}{\partial y_{l}}\right] \frac{\partial}{\partial x_{o}}\left(\gamma_{x 3 n}^{(0)}-\gamma_{3 n}^{0}\right)+\hat{f}_{0}=0
$$

$$
\begin{aligned}
& f_{0}=-f_{0}+\frac{\partial}{\partial x_{j}}\left[D_{j l}\left(\gamma_{x 3 l}^{(0)}-\gamma_{3 l}^{0}\right)+\tau_{3 j}^{0}\right]+ \\
& +\frac{\partial}{\partial y_{j}}\left[D_{j l} \chi_{3}^{3 n}(y)\right] \frac{\partial}{\partial x_{i}}\left(\gamma_{x 3 n}^{(0)}-\gamma_{3 n}^{0}\right)
\end{aligned}
$$

\section{Constraints from Higher-Order Solutions}

If the expansion is truncated to the second-order term $u_{3}^{(2)}$, its contribution to the $O\left(\varepsilon^{1}\right)$ order equilibrium equation needs also to be considered. The unwanted higher-order term $u_{3}^{(3)}$ in the equation can be eliminated by integrating the complete $O\left(\varepsilon^{1}\right)$ order equilibrium equation over $Y$. We thus have

$$
\begin{aligned}
& \int_{Y}\left\{D_{j l}\left[\chi_{3}^{3 n}(\mathbf{y}) \delta_{l o}+\frac{\partial \psi_{3}^{3 n o}(y)}{\partial y_{1}}\right]\right\} d Y . \\
& \frac{\partial^{2}}{\partial x_{j} \partial x_{o}}\left(\gamma_{x 3 n}^{(0)}\left(u_{3}^{(0)}\right)-\gamma_{3 n}^{0}\right)=0
\end{aligned}
$$

or

$$
\int_{Y} D_{j l} \frac{\partial \psi_{3}^{3 n o}(\mathbf{y})}{\partial y_{1}} d Y=-\int_{Y} D_{j o} \chi_{3}^{3 n}(\mathbf{y}) d Y
$$

\section{$O\left(\varepsilon^{1}\right) \quad$ Equilibrium: Third-Order} Homogenisation

\section{Solution of $u_{3}^{(3)}$}

With the use of (69), (71) and (86), equation (75) becomes

$$
\begin{aligned}
& \frac{\partial \tau_{3 j}^{(1)}}{\partial x_{j}}+\frac{\partial \tau_{3 j}^{(2)}}{\partial y_{j}}=\frac{\partial}{\partial x_{j}} \\
& \left\{D_{j l}\left[\chi_{3}^{3 n}(\mathbf{y}) \frac{\partial}{\partial x_{1}}\left(\gamma_{x 3 n}^{(0)}\left(u_{3}^{(0)}\right)-\gamma_{3 n}^{0}\right)+\frac{\partial u_{3}^{(2)}}{\partial y_{1}}\right]\right\} \\
& +\frac{\partial}{\partial y_{j}}\left\{D_{j l}\left[\frac{\partial u_{3}^{(2)}}{\partial x_{1}}+\frac{\partial u_{3}^{(3)}}{\partial y_{1}}\right]\right\}=0
\end{aligned}
$$


Assume $D_{i j}$ is not explicitly dependent on $\mathbf{x}, u_{k}^{(3)}$ can also be separated into $\mathbf{x}$ - and $\mathbf{y}$-dependent terms

$$
u_{3}^{(3)}=\phi_{3}^{3 n o p}(y) \frac{\partial^{2}}{\partial x_{p} \partial x_{o}}\left(\gamma_{x 3 n}^{(0)}-\gamma_{3 n}^{0}\right)
$$

(95) can be rewritten into the following equations

$$
\frac{\partial}{\partial y_{j}}\left(D_{j l} \frac{\partial u_{3}^{(3)}}{\partial y_{1}}\right)-f_{0}^{(3)}=0
$$

where

$$
\begin{aligned}
& f_{0}^{(3)}=-\frac{\partial}{\partial x_{j}} \\
& \left\{D_{j l}\left[\chi_{3}^{3 n}(\mathbf{y}) \frac{\partial}{\partial x_{1}}\left(\gamma_{x 3 n}^{(0)}-\gamma_{3 n}^{0}\right)+\frac{\partial u_{3}^{(2)}}{\partial y_{1}}\right]\right\} \\
& -\frac{\partial}{\partial y_{j}}\left\{D_{j l} \frac{\partial u_{3}^{(2)}}{\partial x_{1}}\right\}
\end{aligned}
$$

\section{Constraints from Higher-Order Terms}

For the same reason as in solving for $u_{3}^{(2)}$, we need also to consider the $O\left(\varepsilon^{2}\right)$ order equilibrium equation. Again, integration of the complete $O\left(\varepsilon^{2}\right)$ order equilibrium equation over $Y$ gives

$$
\begin{aligned}
& \frac{\partial}{\partial x_{j} Y} \\
& \left\{\int_{d Y=0}^{3 n o}\left(\psi_{3}^{3 n}(\mathbf{y}) \frac{\partial^{2}}{\partial x_{1} \partial x_{o}}\left(\gamma_{x 3 n}^{(0)}\left(u_{3}^{(0)}\right)-\gamma_{3 n}^{0}\right)+\frac{\partial u_{3}^{(3)}}{\partial y_{1}}\right]\right\}
\end{aligned}
$$

or

$$
\int_{Y} D_{j l} \frac{\partial \phi_{3}^{3 n o p}}{\partial y_{1}} d Y=-\int_{Y} D_{j p} \psi_{3}^{3 n o}(\mathbf{y}) d Y
$$

Obviously, terms higher than the third-order can be solved in a similar way. The controlling equations for the $p$ th order $(p \geq 3)$ displacements are

$$
\frac{\partial}{\partial y_{j}}\left(D_{j l} \frac{\partial u_{3}^{(p)}}{\partial y_{1}}\right)-f_{0}^{(p)}=0
$$

$$
\begin{aligned}
f_{0}^{(p)}= & -\frac{\partial}{\partial x_{j}}\left\{D_{j l}\left[\frac{\partial u_{3}^{(p-2)}}{\partial x_{1}}+\frac{\partial u_{3}^{(p-1)}}{\partial y_{1}}\right]\right\} \\
& -\frac{\partial}{\partial x_{j}}\left\{D_{j l} \frac{\partial u_{3}^{(p-1)}}{\partial x_{1}}\right\}
\end{aligned}
$$

with constraints from higher order terms

$$
\int_{Y} D_{j l}\left(\frac{\partial u_{3}^{(p-1)}}{\partial x_{1}}+\frac{\partial u_{3}^{(p)}}{\partial y_{1}}\right) d Y=0
$$

However, in the numerical implementation, although it is only required to solve a second-order equilibrium equation on the RUC (cf. (91) and (97)), it is actually limited by the requirement of the higherorder derivatives of the solution $u_{3}^{(0)}$ at the macro scale (cf. (92) and (98)).

\section{Solution of Equilibrium Equations from Homogenisation}

To solve the torsion or bending of composite shafts by the homogenisation method, we will first solve for $\chi_{3}^{3 j}(y)$ from equation (83) assuming it to be a $Y$ periodic function defined in $Y$. The effective material properties $\bar{D}_{j k}$ are given by (90). We then solve the homogeneous St Venant torsion or bending problem (89) and obtain the macroscopic fields: warping displacement $u_{3}^{(0)}$, strains $\gamma_{x 3 j}^{(0)}$ and stresses (given by $\left.\bar{D}_{j k} \gamma_{x 3 i}^{(0)}\right)$. If the distribution of the microscopic fields in the neighborhood of point $x$ is of interest, $u_{3}^{(1)}(82)$ can then be obtained from $\chi_{l}^{k l}(\mathbf{y})$ and $u_{3}^{(0)}$. $u_{3}^{(2)}$ can next be solved from (91) with constraints (93) or (94); $u_{3}^{(3)}$ can be solved from (97) with constraints (99) or (100). Higher-order displacement terms can be solved in a similar way. The strains $\gamma_{3 j}$ and stresses $\tau_{3 j}$ can be calculated from (69) and (71), respectively. Equations (89), (91), (97) and (101) are standard second order partial differential equations in solid mechanics. They can be solved in a similar way. However, for a problem defined on the unit cell $Y$ the periodic boundary conditions and constraints 
from higher-order equilibrium should be enforced appropriately.

As all the equilibrium equations can be solved similarly, only equation (83) is discussed. Corresponding to (83), the virtual work principle states that

$$
\int_{Y} \delta \chi_{k}^{3 k} \frac{\partial}{\partial y_{j}}\left(D_{j l} \frac{\partial \chi_{3}^{3 k}}{\partial y_{1}}\right) d Y+\int_{Y} \delta \chi_{k}^{3 k} \frac{\partial D_{j k}}{\partial y_{j}} d Y=0
$$

or

$$
\int_{Y} \frac{\partial \delta \chi_{3}^{3 k}}{\partial y_{j}} D_{j l} \frac{\partial \chi_{3}^{3 k}}{\partial y_{1}} d Y+\int_{Y} \frac{\partial \delta \chi_{3}^{3 k}}{\partial y_{j}} D_{j k} d Y=0
$$

where $\delta \chi_{3}^{3 k}$ are arbitrary $Y$-periodic functions defined in the unit cell $Y$.

It is easy to prove that (104) is the first order variation of the following potential functional

$$
\begin{aligned}
\Pi_{p}\left(\chi_{3}^{3 k}\right)= & \int_{Y} \frac{1}{2} \frac{\partial \chi_{k}^{3 k}}{\partial y_{j}} D_{j l} \frac{\partial \chi_{k}^{3 k}}{\partial y_{i}} d Y \\
& +\int_{Y} \frac{\partial \chi_{k}^{3 k}}{\partial y_{j}} D_{j k} d Y
\end{aligned}
$$

If we define the strain

$$
\tilde{\gamma}_{3 l}^{k}=\frac{\partial \chi_{3}^{3 k}}{\partial y_{i}}
$$

and the stress

$$
\tilde{\tau}_{3 j}^{k}=D_{j l} \tilde{\gamma}_{3 l}^{k} \text { so that } \tilde{\gamma}_{3 l}^{k}=C_{i j} \tilde{\tau}_{3 j}^{k}
$$

which are Y-periodic functions in the unit cell, we have a 2-field Hellinger-Reissner functional

$$
\begin{aligned}
& \Pi_{H R}\left(\chi_{3}^{3 k}, \tilde{\tau}_{3 j}^{k}\right)=\int_{Y}\left[-\frac{1}{2} \tilde{\tau}_{3 j}^{k} C_{i j} \tilde{\tau}_{3 j}^{k}\right. \\
& \left.+\tilde{\tau}_{3 i}^{k} \frac{\partial \chi_{3}^{3 k}}{\partial y_{i}}+D_{j k} \frac{\partial \chi_{3}^{3 k}}{\partial y_{j}}\right] d Y
\end{aligned}
$$

By relaxing the compatibility condition in the potential (105) or by employing Legendre transformation on the Hellinger-Reissner (108) one arrives at the 3-field $\mathrm{Hu}$-Washizu functional

$$
\begin{aligned}
& \Pi_{H W}\left(\chi_{3}^{3 k}, \tilde{\gamma}_{3 j}^{k}, \tilde{\tau}_{3 j}^{k}\right)=\int_{Y}\left[-\frac{1}{2} \tilde{\gamma}_{3 i}^{k} D_{i j} \tilde{\gamma}_{3 j}^{k}\right. \\
& \left.-\tilde{\tau}_{3 i}^{k}\left(\tilde{\gamma}_{3 j}^{k}-\frac{\partial \chi_{3}^{3 k}}{\partial y_{i}}\right)+D_{j k} \frac{\partial \chi_{3}^{3 k}}{\partial y_{j}}\right] d Y
\end{aligned}
$$

The general isoparametric compatible elements are not satisfactory because of the gradients of $\chi_{3}^{3 k}$ that appear in (90) in the evaluation of the homogenised material properties. The shape functions in (12)-(17) can be adapted to interpolate $\chi_{3}^{3 k}$ to formulate an equivalent displacement-incompatible element from the potential functional (105), or enhanced-strain element from the Hu-Washizu functional (109); adaption of the interpolation (13)(15) for $\chi_{3}^{3 k}$ and (34) for $\tilde{\tau}_{3 i}^{k}$ can formulate an equivalent hybrid stress element from the HellingerReissner functional (108).

Strictly speaking, solution of the Y-periodic functions on the RVE needs to satisfy the periodic conditions on the boundary, including the primary compatible displacement functions as well as the incompatible displacement/strain-enhanced terms for incompatible/strain-enhanced elements and assumed stresses for hybrid stress elements. The periodic constraints on compatible displacements can be imposed by a penalty function approach (Karihaloo et al. 2001; Xiao and Karihaloo, 2009), which can also be used to enforce the constraints from higher order terms; imposition of such constraints on incompatible displacements, enhanced-strains or stresses is difficult. However, numerical results (Feng and Wu, 2001; Karihaloo et al. 2001; Sun et al. 2001) show that neglecting the periodic constraints on these element internal fields does not deteriorate the solution.

\section{Numerical Results}

The performance of the incompatible/strain-enhanced element is almost identical to the hybrid stress element, we therefore only give the results from one of them in the following examples.

\section{Torsion of Compound Square Shaft}

Torsion of the compound square shaft of unit side 
length composed of two equal in homogeneous regions shown in Fig. 4 with unit angle of twist per unit length is analysed. As in Ely and Zienkiewicz (1960) and Jog and Mokashi (2014), $G_{1}$ is fixed at 1 with $E_{1}=$ 2.5 and $\nu_{1}=0.25$, three different values of $G_{2} / G_{1}=1$, 2 and $3\left(v_{2}=v_{1}\right)$ are considered. The cross-section is discretised into 88 four-node elements following Jog and Mokashi (2014); for the case $G_{2} / G_{1}=3$ a fine discretisation of $40 \times 40$ is also used. The torsional rigidities obtained by the hybrid stress element are compared against the analytical solution of Muskhelishvilli (1953), the relaxation finite difference method of Ely and Zienkiewicz (1960), and the 4node element solution of Jog and Mokashi (2014) in Table 1. For a homogeneous shaft, the solution of Muskhelishvilli (1953) is identical to Timoshenko and Goodier (1969), as expected. The results from the hybrid stress element are the most accurate. The $\mathrm{x}$ coordinate of the centroid and effective shear modulus are given in Table 2 . The warping at $\mathrm{A}(-0.5,-0.25)$ and $\mathrm{D}(0.5,-0.25)$ relative to the centroid, and shear stresses at $\mathrm{B}(-0.5,0)$ and $\mathrm{C}(0.5,0)$ (Fig. 4$)$ from the hybrid stress element are given in Table 3 . For a homogeneous shaft, $G_{2}=G_{1}$, the warping and shear stresses agree reasonably well with the analytical solutions $\varphi_{A}=-\varphi_{D}=0.0347, \tau_{C y z}=-\tau_{B y z}=0.6753$ given by Timoshenko and Goodier (1969) despite the coarse mesh adopted. For the case $G_{2} / G_{1}=3$, the results from the coarse mesh agree well with those from the fine mesh.

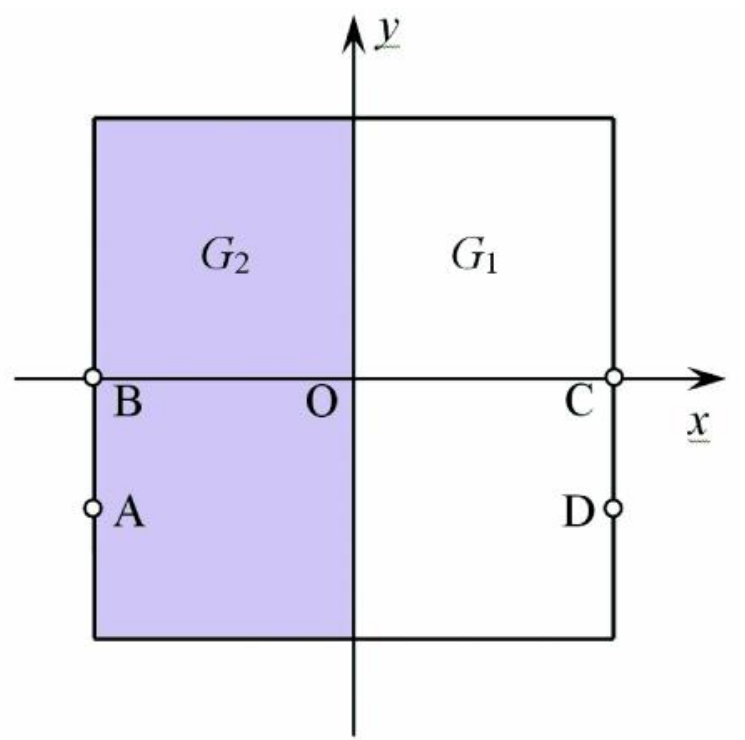

Fig. 4: Square cross-section of unit side length composed of two equal inhomogeneous regions
Table 1: Torsional rigidity for compound square shaft

\begin{tabular}{lllll}
\hline$G_{2} / G_{1}$ & $\begin{array}{l}\text { Muskheli- } \\
\text { shvilli } \\
(1953)\end{array}$ & $\begin{array}{l}\text { Ely and } \\
\text { Zienkiewicz } \\
(1960)\end{array}$ & $\begin{array}{l}\text { Jog and } \\
\text { Mokashi } \\
(2014)\end{array}$ & $\begin{array}{l}\text { Present hybrid } \\
\text { element }\end{array}$ \\
\hline 1 & 0.1406 & 0.1388 & 0.1425 & 0.1398 \\
2 & 0.1970 & 0.1941 & 0.1997 & 0.1957 \\
3 & 0.2394 & 0.2358 & 0.2430 & $0.2377\left(0.2396^{*}\right)$ \\
\hline
\end{tabular}

*From refined discretisation 40x40

Table 2: $x$-coordinate of centroid and effective shear modulus

\begin{tabular}{lccc}
\hline$G_{2} / G_{1}$ & Discretisation & $\mathrm{x}_{\mathrm{c}}$ & $\mathrm{G}_{\mathrm{eff}}$ \\
\hline 1 & $8 \times 8$ & 0 & 1 \\
2 & $8 \times 8$ & & 1.3479 \\
3 & $8 \times 8$ & -0.125 & 1.5647 \\
& $40 \times 40$ & -0.125 & 1.5628 \\
\hline
\end{tabular}

Table 3: Warping and shear stresses for torsion of compound square shaft

\begin{tabular}{lllrrr}
\hline$G_{2} / G_{1}$ & Discretisation & $\varphi$ at $\mathrm{A}$ & $\tau_{y z}$ at B & $\varphi$ at D & $\tau_{y z}$ at $\mathrm{C}$ \\
\hline 1 & $8 \times 8$ & 0.03532 & -0.6691 & -0.03532 & 0.6691 \\
2 & $8 \times 8$ & 0.04044 & -1.2023 & -0.03020 & 0.7370 \\
3 & $8 \times 8$ & 0.04300 & -1.7016 & -0.02764 & 0.7709 \\
& $40 \times 40$ & 0.04224 & -1.7102 & -0.02728 & 0.7801 \\
\hline
\end{tabular}

\section{Bending of Compound Square Shaft}

We now consider torsion-less bending of the above compound square shaft. The shear stresses given by the hybrid stress element at the centre are given in Table 4. Jump in the tangential stresses is noticed at the interface between different materials, and increases with the ratio $G_{2} / G_{1}$. For a homogeneous shaft, $G_{2}=G_{1}$, the analytical solution is $\tau_{x z}=1.4104$ for $Q_{x}=1$ and $Q_{y}=0$, and $=1.4104$ for $Q_{x}=0$ and $Q_{y}$ $=1$ (Timoshenko and Goodier, 1969); the solution of the hybrid stress element agrees favourably with the analytical solution despite the coarse mesh adopted. In order to verify the accuracy of the hybrid stress element for inhomogeneous shafts, we analyse a cantilever of length 10 under transverse loading at the free end. The cantilever is divided into $16 \times 16$ (section) x 40 (length) 20-noded hexahedral elements. The results at the middle section are also included in 


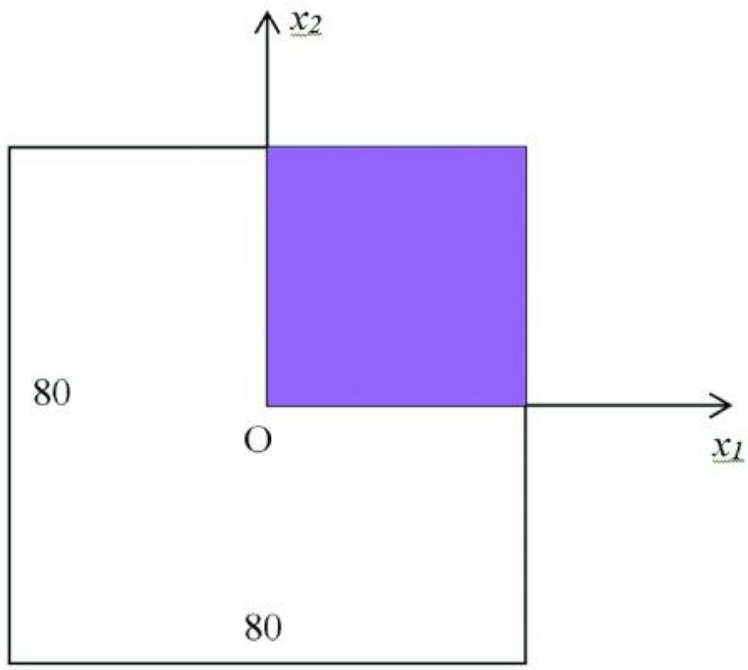

(A)

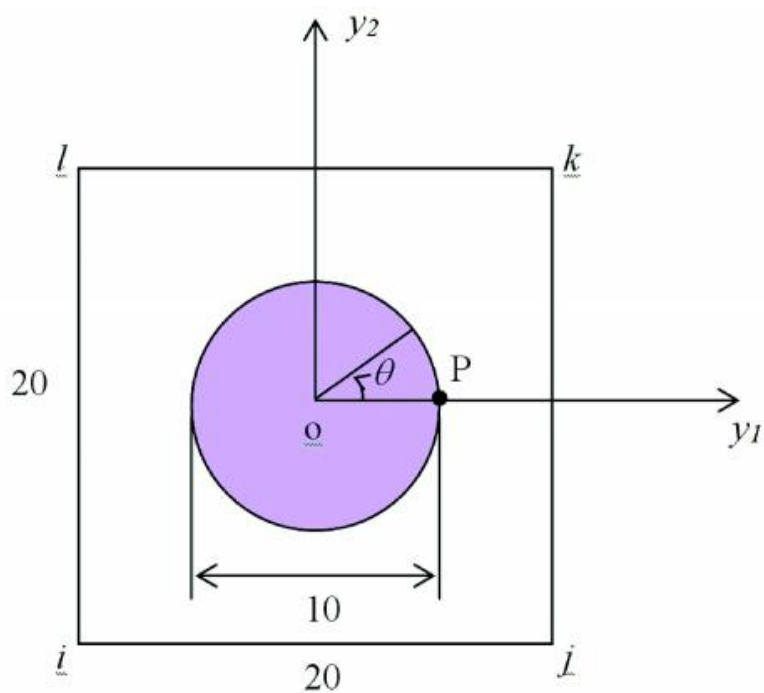

(B)

Fig. 5: Geometry of a composite shaft of square profile (A) and RUC (B)

Table 4, agreeing reasonably with the hybrid stress element with the fine discretisation.

\section{Torsion of a Square Composite Shaft}

The example for the torsion of a composite shaft analysed by Karihaloo et al. (2001) is adopted to illustrate the first order homogenisation method. The composite shaft has a square cross-section (length of side $=80$ ), as shown in Fig. 5(A); the microstructure of the cross-section is locally periodic with a period defined by a RUC shown in Fig. 5(B), i.e. it consists of an isotropic circular fibre of diameter 10 embedded in an isotropic square matrix with side 20. Assume that the scaling factor $\varepsilon=0.25$. The problem is solved in two stages. First, we solve the RUC shown in Fig. 5(B) by using the incompatible/strain-enhanced

Table 4: Shear stresses at centre of compound square shaft under bending

\begin{tabular}{llllll}
\hline$G_{2} / G_{1}$ & Discretisation & \multicolumn{2}{c}{$Q_{x}=1$} & \multicolumn{2}{c}{$Q_{y}=1$} \\
& & left & right & left & right \\
\hline 1 & $8 \times 8$ & 1.3760 & 1.3760 & 1.3760 & 1.3760 \\
2 & $8 \times 8$ & 1.3981 & 1.3024 & 1.8553 & 0.9277 \\
3 & $8 \times 8$ & 1.3916 & 1.2297 & 2.1163 & 0.7054 \\
& $40 \times 40$ & 1.3289 & 1.2912 & 2.1658 & 0.7219 \\
& $1616 \times 40^{\dagger}$ & 1.3077 & 1.3038 & 2.3265 & 0.7755 \\
\hline
\end{tabular}

$\dagger$ 20-noded hexahedral element element to obtain the field $\chi_{3}^{3 k}$ and its derivatives $\frac{\partial}{\partial y_{j}} \chi_{3}^{3 k}$ and calculate the homogenised moduli from (90). Second, we solve the torsion of the square shaft shown in Fig. 5(A) with the obtained homogenised moduli by using the hybrid stress element. Only a quarter of the cross-section, the shaded part shown in Fig. 5(A), is dicretised because of symmetry. The warping displacements are fixed on the axes of symmetry. In this way, we calculate the warping displacement, torsional rigidity and the angle of twist per unit length, as well as the shear stresses and strains. With the results so obtained, we can calculate the first order warping displacement from (82) and the local strain and stress fields from (69) and (71), respectively.

The shaded quarter in Fig. 5(A) is discretised into 400 quadrilateral elements and 441 nodes, as shown in Fig. 6(A). The RUC shown in Fig. 5(B) is discretised into 896 quadrilateral elements and 929 nodes, as shown in Fig. 6(B).

The fibre and the matrix are considered to be isotropic with the shear moduli, $G_{f}=10$ and $G_{m}=1$, respectively. The computed homogenised shear moduli are

$$
\left[\begin{array}{cc}
C_{11} & C_{12} \\
\text { Sym } & C_{22}
\end{array}\right]=\left[\begin{array}{cc}
1.38271 & -0.00138 \\
\text { Sym } & 1.38467
\end{array}\right]
$$


Thus the macroscopic behaviour of the composite shaft is also isotropic.

The isotropic shaft of square cross-section shown in Fig. 5(A) is now analysed with the obtained homogenised shear moduli. One unit of torque is applied on the quarter section with its units being consistent with those of the shear moduli. The computed result for the torsional rigidity $4 \times 1.9927 \mathrm{x}$ $10^{6}$ is very close to the accurate value $7.9856 \times 10^{6}$ obtained from the formula $0.1406 \mathrm{G}(2 \mathrm{~b})^{2}$ where the shear modulus $G=1.38271$, and the length of side of the square cross-section $2 b=80$ in the present example. The distribution of warping displacement, and of normal and tangential shear stresses, which are given by

(A)

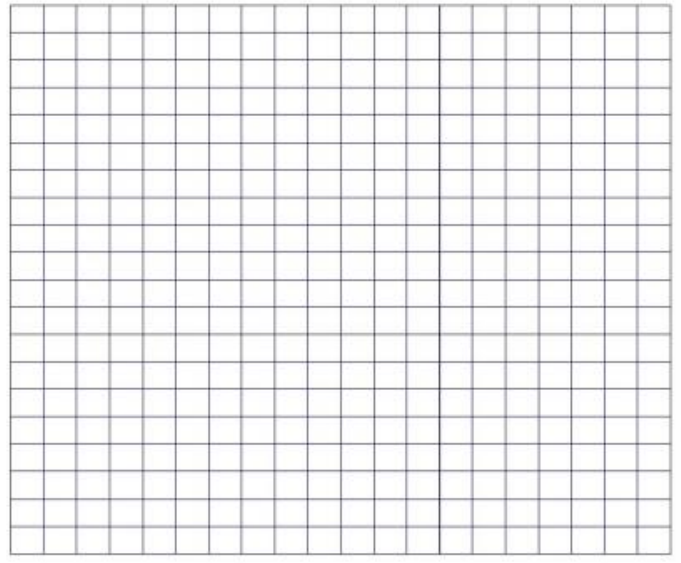

(B)

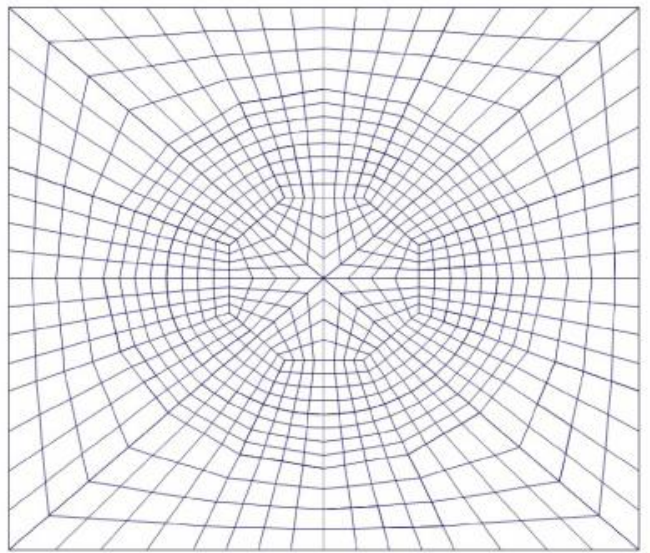

Fig. 6: Discretised meshes used in the computation: (A) Mesh of a quarter of the cross-section shown in Fig. 5(A); (B) Mesh of the RUC shown in Fig. 5(B) where $\theta$ is the angle from the axis $y_{1}$ as shown in Fig. 5(B), along the interface between the fibre and the matrix adjacent to the point with global co-ordinates $\left(x_{1}=30, x_{2}=30\right)$ are plotted in Fig. 7. Filled triangles represent computed data. In Fig. 7(B) and (C), data linked by broken lines represent the results obtained from the matrix side (a line segment represents the distribution of stress within an element), the continuous solid line represents the polynomial fit of the computed

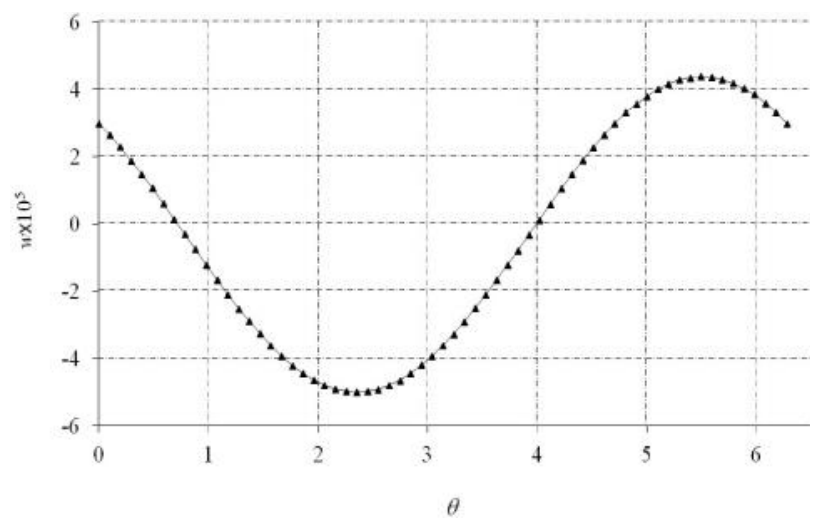

(A)

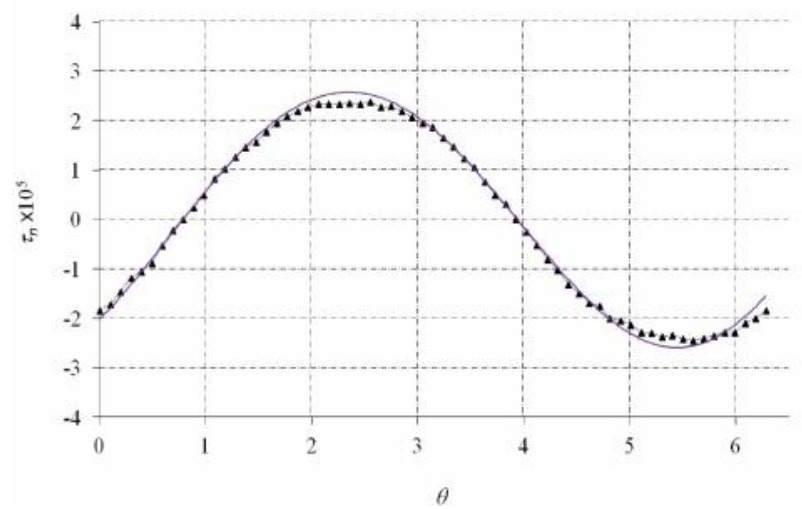

(B)

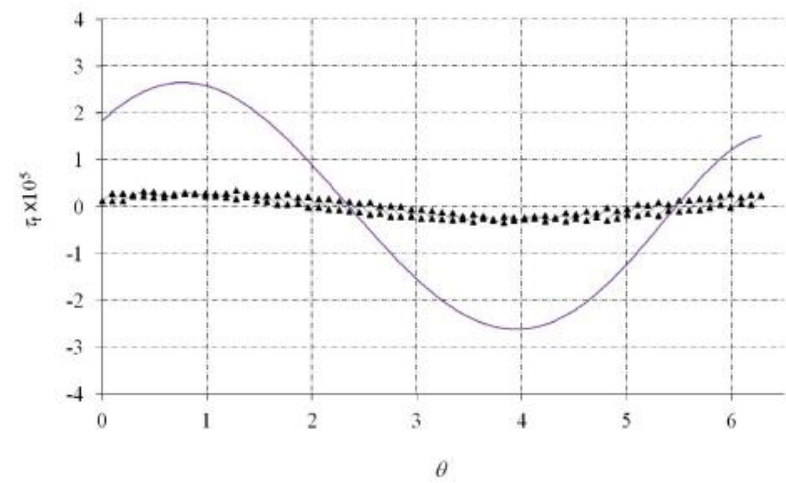

(C)

Fig. 7: Distribution of warping displacement (A), normal shear stress, (B) and tangential shear stress and (C) along the interface from the homogenisation method 
results obtained from the fibre side of the interface since they are not satisfactorily smooth. From Fig. 7, the tangential shear stress $\tau_{t}$ has a significant discontinuity across the interface, while other fields are continuous adjacent to the interface.

\section{Conclusions and Discussion}

Formulation of the St Venant torsion of prismatic composite shafts with properties uniform along the length and piece-wise constant orthotropic across the cross-section is given in terms of the warping function. The principal axes of all constituents of the crosssection are assumed to be parallel. High-performance incompatible, strain-enhanced and hybrid stress elements for solving the warping function are discussed. In particular, a hybrid stress element developed earlier has been improved to avoid the need of a special node numbering pattern for square elements.

Formulation of the St venant bending (or shearing) of Gruttmann et al. (1999) and Gruttmann and Wagner (2001) for prismatic homogeneous isotropic shafts in terms of the warping function has

\section{References}

Barretta R (2013) On Cesàro-Volterra method in orthotropic SaintVenant beam $J$ Elast 112 233-253

Battini J M and Pacoste C (2002) Co-rotational beam elements with warping effects in instability problems Comput Methods Appl Mech Engrg 191 1755-1789

Bensoussan A, Lions J L and Papanicolaou G (2011) Asymptotic analysis for periodic structures American Mathematical Soc

Desai C S (1979) Elementary finite element method Englewood Cliffs: Prentice-Hall

Ely J F and Zienkiewicz O C (1960) Torsion of compound bars - a relaxation solution Int J Mech Sci 1356-365

Feng M L and Wu C C (2001) A study of three-dimensional fourstep braided piezo-ceramic composites by the homogenization method Compos Sci Tech 61 1889-1898

Gruttmann F, Sauer R and Wagner W (1999) Shear stresses in prismatic beams with arbitrary cross-sections Int J Numer Meth Engng 45 865-889

Gruttmann, F, Sauer, R and Wagner, W (2000) Theory and numerics of three dimensional beams with elastoplastic material behaviour Int J Numer Meth Engng 48 1675-1702 been generalised to piece-wise constant orthotropic shafts; the requirement of zero in-plane shear strain over the cross-section, which is implicitly violated by the assumed strain field of Gruttmann et al. (1999) and Gruttmann and Wagner (2001), has been satisfied exactly in the present development. The warping function for bending can be solved by the same elements for the torsional warping function. Unlike Gruttmann et al. (1999) and Gruttmann and Wagner (2001), where the torsional warping function appears in the shear strains of bending and needs to be solved before the solution of bending, the formulation of bending in the present paper is purely in term of the warping function for bending.

In order to solve composite shafts for which the use of a finite element discretisation to represent all the microstructural details is too expensive or even impossible, the first order homogenisation for St Venant torsion (Karihaloo et al., 2001) has been generalised to higher order homogenisation following Xiao and Karihaloo (2009) for both St Venant torsion and bending; a more appropriate method is introduced to consider the constraints from higher order terms.

Gruttmann F and Wagner W (2001) Shear correction factors in Timoshenko's beam theory for arbitrary shaped crosssections Comput Mech 27 199-207

Jog C S and Mokashi I S (2014) A finite element method for the Saint-Venant torsion and bending problems for prismatic beams Comput Struct 135 62-72

Karihaloo B L and Hemp W S (1987) Optimum shapes for given torsional and bending rigidities Proc R Soc Lond A4096777

Karihaloo, B L, Xiao Q Z and Wu C C (2001) Homogenizationbased multivariable element method for pure torsion of composite shafts Comput Struct 79 1645-1660

Kosmatka J B and Dong S B (1991) Saint-Venant solutions for prismatic anisotropic beams Int J Solids Struct 28 917-938

Kourtis L C, Kesari H, Carter D R and Beaupré G S (2009) Transverse and torsional shear stresses in prismatic bodies having inhomogeneous material properties using a new 2D stress function J Mech Mater Struct 4 659-674

Lekhnitskii S G (1963) Theory of Elasticity of an Anisotropic Elastic Body. Holden-Day

Mason W E and Herrmann L R (1968) Elastic shear analysis of general prismatic beams J Eng Mech Div ASCE 94 965986 
Mokos V G and Sapountzakis E J (2005) A BEM solution to transverse shear loading of composite beams Int J Solids Struct 42 3261-3287

Muskhelishvilli N I (1953) Some basic problems of the mathematical theory of elasticity Noordhoff, Groningen

Pian T H H and Sumihara K (1984) Rational approach for assumed stress finite elements Int J Numer Meth Engng 20 16851695

Pian T H H and Wu C C (2006) Hybrid and incompatible finite element methods $\mathrm{CRC}$ Press

Pilkey W D (2002) Analysis and design of elastic beams: Computational methods John Wiley \& Sons

Romano G, Barretta A and Barretta R (2012) On torsion and shear of Saint-Venant beams Eur J Mech A/Solids 35 47-60

Simo J C and Rifai M S (1990) A class of mixed assumed strain methods and the method of incompatible modes Int J Numer Meth Engng 29 1595-1638

Simo J C and Vu-Quoc L (1991) A geometrically-exact rod model incorporating shear and torsion-warping deformation Int $J$ Solids Struct 27 371-393
Sun H Y, Di S L, Zhang N and Wu C C (2001) Micromechanics of composite materials using multivariable finite element method and homogenization theory Int J Solids Struct $\mathbf{3 8}$ 3007-3020

Timoshenko S P and Goodier J N (1969) Theory of elasticity (3rd edition) McGraw-Hill

Wagner W and Gruttmann F (2001) Finite element analysis of Saint-Venant torsion problem with exact integration of the elastic-plastic constitutive equations Comput Methods Appl Mech Engrg 190 3831-3848

Xiao Q Z and Karihaloo B L (2009) Two-scale asymptotic homogenisation-based finite element analysis of composite materials In: Multiscale Modeling in Solid Mechanics: Computational Approaches (Eds: Galvanetto U and Aliabadi M H F) pp 43-100, World Scientific

Xiao Q Z, Karihaloo B L, Li Z R and Williams F W (1999) An improved hybrid-stress element approach to torsion of shafts Comput Struct 71 535-563

Yuan Z, Wu C C and Li H (2003) Homogenization-based topology design for pure torsion of composite shafts Acta Mechanica Sinica 19 241-246. 
